\title{
Reverse Genetics of Coronaviruses Using Vaccinia Virus Vectors
}

\author{
V. Thiel ${ }^{1}(\bowtie) \cdot$ S. G. Siddell ${ }^{2}$ \\ ${ }^{1}$ Research Department, Cantonal Hospital St. Gallen, St. Gallen, Switzerland \\ volker.thiel@kssg.ch \\ ${ }^{2}$ Department of Pathology and Microbiology, School of Medical and Veterinary \\ Sciences, University of Bristol, Bristol, UK
}

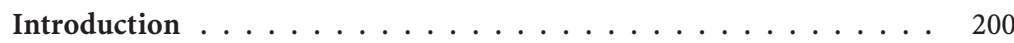

2 The Use of Vaccinia Virus as a Vector for Coronavirus cDNA . . . . . . 202

2.1 Cloning of Full-Length Coronavirus cDNA into the

Vaccinia Virus Genome . . . . . . . . . . . . . . . . . 202

$2.2 \quad$ Mutagenesis of Cloned Coronavirus cDNA . . . . . . . . . . . . . . 204

2.3 Rescue of Recombinant Coronaviruses . . . . . . . . . . . . . . . . . 206

2.3.1 Rescue of Recombinant Coronaviruses Using Full-Length

In Vitro Transcripts . . . . . . . . . . . . . . . . . . . . . 207

2.3.2 Rescue of Recombinant Coronavirus Using Full-Length cDNA . . . . . . 209

2.3.3 Expression of the Nucleocapsid Protein Facilitates the Rescue of Recombinant Coronaviruses . . . . . . . . . . . . . 210

3 Recombinant Coronaviruses . . . . . . . . . . . . . . . 211

3.1 Analysis of HCoV 229E Replicase Polyprotein Processing . . . . . . . . 212

$3.2 \quad$ Analysis of IBV Spike Chimeras . . . . . . . . . . . . . . . . . . . . 215

3.3 Recombinant MHV Is Fully Pathogenic in Mice . . . . . . . . . . . . 215

$4 \quad$ Generation of Replicon RNAs . . . . . . . . . . . . . . . . . . . 217

4.1 Replicase Gene Products Suffice for Discontinuous Transcription . . . . 217

4.2 Generation of Autonomously Replicating RNAs . . . . . . . . . . . . 219

5 Development of Coronavirus-Based Multigene Vectors . . . . . . . 221

5.1 Multigene Expression Using Coronavirus-Based Vectors . . . . . . . . . 222

5.2 Coronavirus-Based Vectors as Potential Vaccines . . . . . . . . . . . . 223

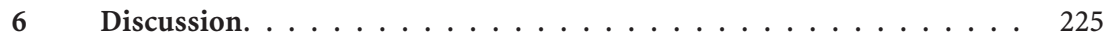

References. . . . . . . . . . . . . . . . . . . 226

Abstract In this article, we describe the reverse genetic system that is based on the use of vaccinia virus cloning vectors. This system represents a generic approach to coronavirus reverse genetics and was first described for the generation of recombinant human coronavirus $229 \mathrm{E}$ representing a group I coronavirus. Subsequently, the same approach has been used to generate recombinant avian infectious bronchitis coronavirus 
and, recently, recombinant mouse hepatitis virus, representing group III and group II coronaviruses, respectively. We describe how vaccinia virus-mediated homologous recombination can be used to introduce specific mutations into the coronavirus genomic cDNA during its propagation in vaccinia virus and how recombinant coronaviruses can be isolated. Finally, we describe how the coronavirus reverse genetic system has now been extended to the generation of coronavirus replicon RNAs.

\section{1}

\section{Introduction}

The development of systems for manipulating the coronavirus genome using traditional reverse genetic approaches has presented a considerable technological challenge because of both the genome size and the instability of specific coronavirus cDNA sequences in bacterial systems. However, recently, reverse genetic systems for a number of coronaviruses have been established using non-traditional approaches which are based on the use of bacterial artificial chromosomes (Almazan et al. 2000 ), the in vitro ligation of coronavirus cDNA fragments (Yount et al. 2000 ) and the use of vaccinia virus as a vector for the propagation of coronavirus genomic cDNAs (Thiel et al. 2001a). With the systems now available, it is possible to genetically modify coronavirus genomes at will. Recombinant viruses with gene inactivations, deletions or attenuating modifications can be generated and used to study the role of specific gene products in viral replication or pathogenesis. Genetically attenuated viruses can be produced which are potential vaccine candidates and modified coronavirus genomes have been developed as eukaryotic, multigene expression vectors (Thiel et al. 2003). In this article, we shall describe the reverse genetic system that is based on the use of vaccinia virus cloning vectors. This system represents a generic approach to coronavirus reverse genetics and was first described for the generation of recombinant human coronavirus 229E ( $\mathrm{HCoV} 229 \mathrm{E}$ )(Thiel et al. 2001a), representing a group I coronavirus. Subsequently, we have used the same approach to generate recombinant avian infectious bronchitis coronavirus (IBV) (Casais et al. 2001) and, recently, recombinant mouse hepatitis virus (MHV-A59) (Coley et al., manuscript in preparation), representing group III and group II coronaviruses, respectively.

The basic strategy for the generation of recombinant coronaviruses can be divided into three phases.

The Assembly of a Full-Length Coronavirus Genomic cDNA. This normally involves the generation of numerous subgenomic cDNA fragments 
that are either amplified as bacterial plasmid DNA or prepared in large amounts by preparative reverse-transcriptase polymerase chain reaction (RT-PCR). The cDNAs are then ligated sequentially, in vitro, to produce a small number of cDNAs which encompass the entire genome. The specific ligation strategy is dictated by the sequence of the coronavirus in question, but a common feature is the use of convenient, naturally occurring or engineered restriction sites, especially if they cleave, for example, interrupted palindromic sequences. It is also necessary to modify the cDNAs which represent the $5^{\prime}$ and $3^{\prime}$ ends of the coronavirus genome. Normally, a transcription promoter sequence for the bacteriophage T7 RNA polymerase is positioned upstream the coronavirus genome and a (unique) restriction site, followed by the hepatitis $\delta$ ribozyme is placed downstream of the poly(A) tail of the coronavirus genome. The terminal cDNA constructs must also have appropriate EagI or Bsp120I restriction sites to facilitate cloning into a unique Not $\mathrm{I}$ restriction site present in the genomic DNA of vaccinia virus, strain vNotI/tk.

The Cloning and Propagation of the Coronavirus Genomic cDNA in Vaccinia Virus Vectors. The next stage is to ligate, in vitro, the coronavirus cDNA fragments and the long and short arms of NotI-cleaved vNotI/tk genomic DNA (Merchlinsky and Moss 1992). This ligation is done in the presence of NotI to prevent religation of the vaccinia virus DNA. Subsequently, the ligation reaction is transfected into mammalian cells which have been previously infected with fowlpox virus. Recombinant vaccinia virus, the genome of which includes a full-length copy of the coronavirus genome, is rescued.

Rescue of Recombinant Coronaviruses. Essentially, recombinant coronaviruses are rescued by generating genomic-length RNA transcripts from the coronavirus component of the recombinant vaccinia virus DNA template. These transcripts are then transfected into permissive cells. The transcription reaction is normally done in vitro, but it is also possible to rescue recombinant coronaviruses via the transcription of template DNA in the permissive cell itself. This requires the introduction of non-infectious (i.e. restriction enzyme digested) recombinant vaccinia virus DNA and a source of bacteriophage T7 RNA polymerase, normally a recombinant fowlpox virus, into the permissive cell. Also, as will be described below, we have found that the ability to rescue recombinant coronaviruses is significantly enhanced by (but not dependent on) the 
directed expression of the coronavirus nucleocapsid protein in the transfected cells.

In addition to these basic concepts, this article will describe how vaccinia virus-mediated homologous recombination can be used to introduce specific mutations into the coronavirus genomic cDNA during its propagation in vaccinia virus. Once an infectious coronavirus cDNA has been obtained, this element of the reverse genetic approach is actually the rate-limiting step. It is, therefore, imperative that a rapid and easy procedure is available. Finally, we shall describe how the coronavirus reverse genetic system has now been extended to the generation of coronavirus replicon RNAs.

\section{2}

\section{The Use of Vaccinia Virus as a Vector for Coronavirus cDNA}

The first use of vaccinia virus as a cloning vector for full-length coronavirus cDNA was described for the human coronavirus 229E (HCoV 229E) system. Vaccinia virus vectors were chosen for several reasons. First, poxvirus vectors are suitable for the cloning of large cDNA. It has been shown that they have the capacity to accept at least $26 \mathrm{~kb}$ of foreign sequence (Smith and Moss 1983), and recombinant vaccinia virus genomes of this size are stable, infectious and replicate in tissue culture to the same titre as non-recombinant virus. Second, vaccinia virus vectors have been developed which are designed for the insertion of foreign DNA by in vitro ligation (Merchlinsky and Moss 1992). This obviates the need for plasmid intermediates carrying the entire cDNA insert. Third, the cloned cDNA insert should be accessible to mutagenesis by vaccinia virus-mediated homologous recombination. Finally, conventional cloning strategies based on procaryotic cloning systems (e.g. plasmid vectors, bacterial artificial chromosomes or bacteriophage lambda vectors) were not applicable to the stable propagation of full-length HCoV 229E cDNA.

\section{1}

\section{Cloning of Full-Length Coronavirus CDNA into the Vaccinia Virus Genome}

As outlined above, the overall strategy to insert full-length coronavirus cDNA fragments into the vaccinia virus genome involves two steps. First, the full-length coronavirus cDNA is assembled by in vitro ligation using 
multiple cDNAs representing the entire coronavirus genomic RNA. Second, the vaccinia virus vNotI/tk genome is used as a cloning vector to insert the full-length cDNA, again by in vitro ligation.

The assembly of full-length coronavirus cDNAs for HCoV 229E, IBV and, recently, MHV-A59 has involved two, three or four cDNA fragments, respectively. The DNA fragments corresponding to the $5^{\prime}$-end of the coronavirus genomes contained the bacteriophage T7 RNA polymerase promoter sequence and, if not encoded at the $5^{\prime}$-end of the coronavirus genome, one or three additional $\mathrm{G}$ nucleotides which are required for efficient initiation of the in vitro transcription reaction. The cDNA fragment corresponding to the $3^{\prime}$-end of the coronavirus genome contained a synthetic poly(A) stretch comprised of 20-40 nucleotides (nt), followed by a hepatitis delta ribozyme element and a convenient restriction site that can be used to generate so-called run-off transcripts. In order to insert the full-length cDNAs into a single NotI site of the vaccinia virus vNotI/tk vector genome, the cDNA fragments corresponding to the $5^{\prime}$ - and $3^{\prime}$-genomic termini contained the restriction sites EagI or Bsp120I. After cleavage, the resulting DNA ends are compatible with NotI-cleaved vaccinia virus vector DNA.

The insertion of full-length coronavirus cDNA fragments into the vaccinia virus vNotI/tk genome by in vitro ligation required optimization of the procedure. Purified vaccinia virus vNotI/tk genomic DNA fragments which had been cleaved with NotI were found to be poor substrates for in vitro ligation, most probably because of their large size. In contrast, in vitro assembled full-length coronavirus cDNA fragments which had been cleaved with EagI were found to ligate efficiently. Thus, in ligation reactions containing NotI-cleaved vaccinia virus vector DNA and coronavirus cDNA inserts, the ligation products were predominantly comprised of multiple insert fragments. Ligation products comprised of vector arms and insert cDNA fragments were not readily detectable. To resolve this problem, we therefore included the NotI enzyme in the ligation reaction and, using alkaline phosphatase, dephosphorylated the coronavirus insert DNA fragments. As illustrated in Fig. 1, this strategy resulted in the production of detectable amounts of ligation products comprised of two vaccinia virus vector arms and the coronavirus cDNA insert.

To rescue recombinant vaccinia virus clones containing the fulllength coronavirus CDNA the ligation reaction was transfected into CV-1 cells. Because vaccinia virus genomic DNA is not infectious, fowlpox virus has been used as a helper virus (Scheiflinger et al. 1992). Thus CV-1 cells were infected with fowlpox virus before transfection. At 2-3 h after infection/transfection, the cells were collected and transferred with a 


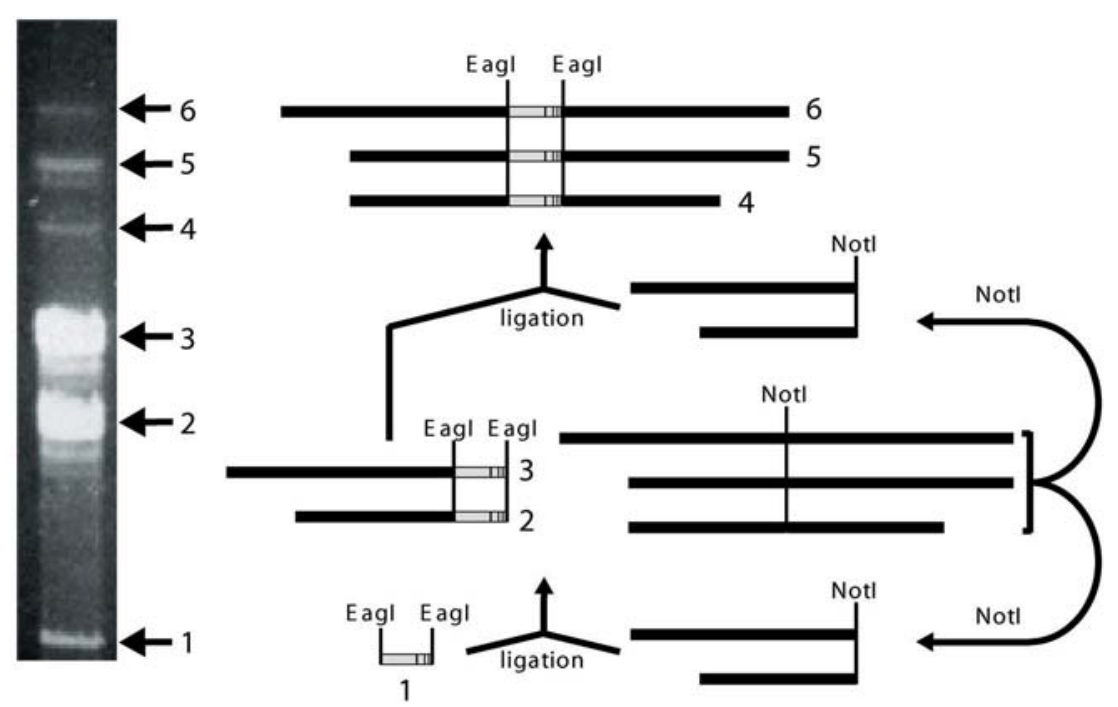

Fig. 1. Cloning of coronavirus cDNA into the vaccinia virus genome. A schematic overview of the optimized ligation reaction using EagI-cleaved and dephosphorylated coronavirus cDNA (1) and NotI-cleaved vaccinia virus genomic DNA is illustrated. The ligation reactions are carried out at $25^{\circ} \mathrm{C}$ in the presence of Not I enzyme. Also shown is a pulse-field gel electrophoresis analysis of the ligation reaction products. Fragments corresponding to the insert cDNA and ligation products comprised of insert cDNA and vaccinia virus DNA $(2,3,4,5$, and 6) are indicated

fivefold excess of fresh CV-1 cells into 96-well plates. During a period of 14 days, virus stocks were collected from 96-well plates displaying cytopathic effect. Because fowlpox virus infection of mammalian cells is abortive, the resulting virus stocks contained exclusively vaccinia viruses. Furthermore, the analysis of genomic DNA of rescued vaccinia viruses by Southern blotting showed that a high percentage of the viruses contained the coronavirus cDNA insert (Thiel et al. 2001a, b).

\section{2}

\section{Mutagenesis of Cloned Coronavirus cDNA}

One major advantage of using vaccinia virus as a cloning vector is that the cloned coronavirus CDNA is amenable to site-directed mutagenesis using vaccinia virus-mediated homologous recombination (Ball 1987). We will show one example to demonstrate the ease of using vaccinia virus-mediated recombination to genetically modify coronavirus cDNA inserts. 

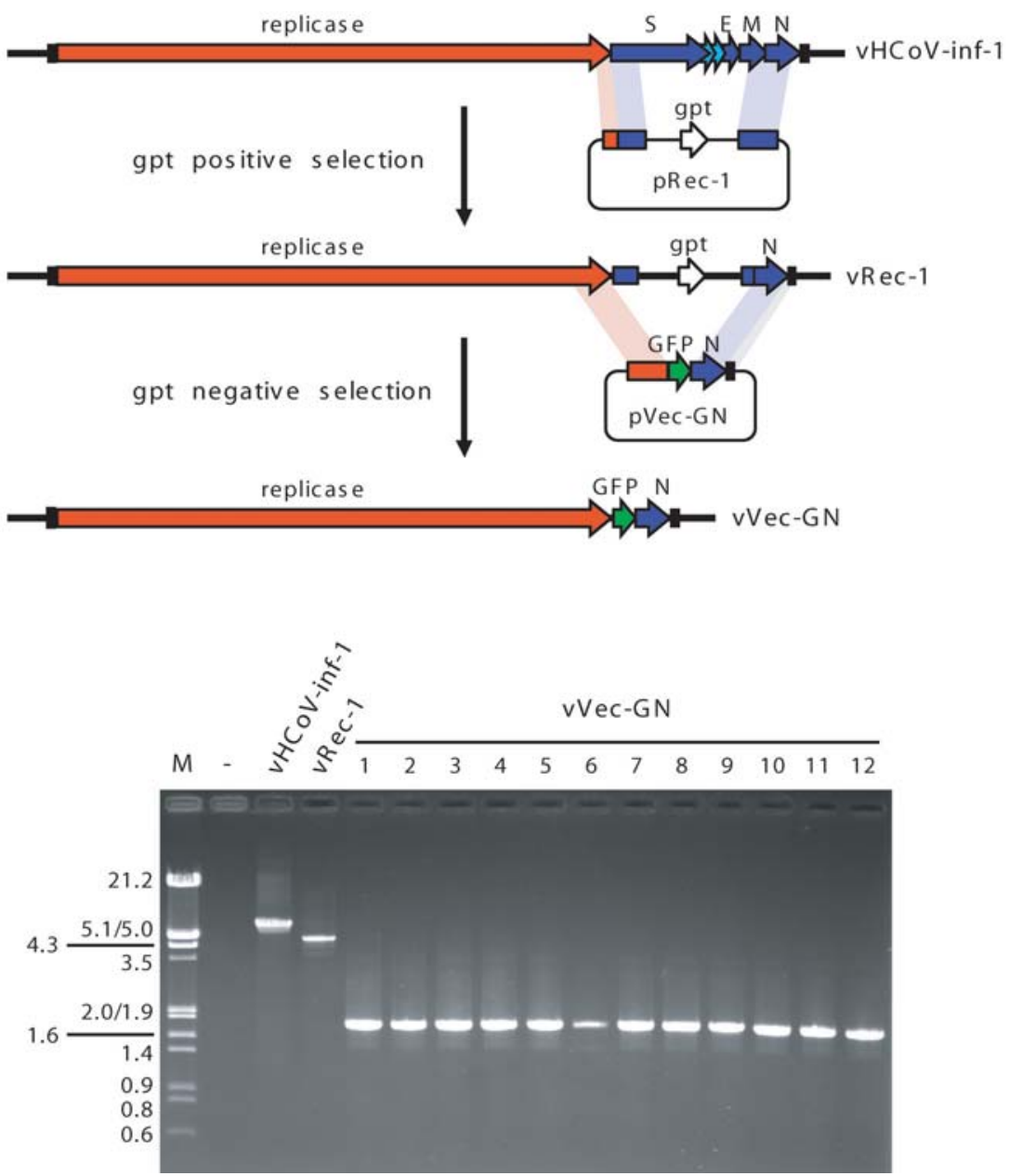

Fig. 2. Mutagenesis of cloned coronavirus cDNA by vaccinia virus-mediated homologous recombination. The generation of the recombinant vaccinia virus vVec-GN containing a modified HCoV 229E genome is illustrated (see text for details). Also shown is a PCR analysis of the region in which the homologous recombination took place within the genomes of the parental vaccinia virus clone vHCoV-inf- 1 , the intermediate vaccinia virus clone vRec-1 and the recombinant vaccinia virus clone vVecGN. Lanes 1-12 show 12 randomly picked recombinant vaccinia virus plaques obtained after the gpt-negative selection, indicating the 100\% recovery of desired genotypes 
A region corresponding to $\mathrm{HCoV}$ nt 20,569-25,653, which lies within the full-length HCoV 229E cDNA insert, has been replaced by the gene encoding the green fluorescent protein (GFP). This results in a recombinant vaccinia virus clone designated vVec-GN. The overall strategy of vVec-GN construction is illustrated in Fig. 2. The procedure is based on using the $E$. coli guanine-phosphoribosyl transferase gene (gpt) as both a positive and a negative selection marker. First, a region corresponding to nt 21,146-24,200 of the HCoV 229E genome was replaced by the $E$. coli gpt gene. To do this, we transfected vHCoV-inf-1-infected CV-1 cells with a plasmid DNA containing the E. coli gpt gene located downstream of a vaccinia virus promoter and flanked by $\mathrm{HCoV} 229 \mathrm{E}$ sequences (nt 19,601-21,145 and nt 24,201-25,874) that facilitate recombination. Two days after infection/transfection, a vaccinia virus stock was prepared. To isolate gpt-containing vaccinia viruses, three rounds of plaque purification were done under gpt-positive selection on CV-1 cells in the presence of mycophenolic acid $(25 \mu \mathrm{g} / \mathrm{ml})$, xanthine $(250 \mu \mathrm{g} / \mathrm{ml})$ and hypoxanthine $(15 \mu \mathrm{g} / \mathrm{ml})$. A recombinant vaccinia virus, designated vRec-1, which contained the E. coli gpt gene at the expected position, could be easily identified by PCR and Southern blot analysis. In a second step, vRec-1 was used to replace the E. coli gpt gene by the GFP gene. CV-1 cells were infected with vRec-1 and transfected with a plasmid DNA encoding the GFP gene flanked by $\mathrm{HCoV}$ sequences (nt 19,485-20,568 and nt 25,654-27,273). Again, after 2 days we prepared a vaccinia virus stock from the infected/transfected cells and did three rounds of plaque purification. However, this time we used HeLa-D980R cells and conditions which allow for the selection of vaccinia viruses that have lost the expression of gpt (Kerr and Smith 1991). A PCR analysis of 12 vaccinia virus clones (Fig. 2) demonstrates that, in each case, vaccinia virus-mediated homologous recombination has taken place at the expected position within the cloned HCoV 229E insert DNA. One of the recombinant vaccinia virus clones was subjected to sequencing analysis of the region where the vaccinia virus-mediated recombination had occurred, and the results revealed that vaccinia virus-mediated recombination is precise at the nucleotide level.

\section{3}

\section{Rescue of Recombinant Coronaviruses}

Two strategies have been reported for the rescue of recombinant coronaviruses from full-length cDNA cloned in vaccinia virus vectors. Initially, recombinant $\mathrm{HCoV} 229 \mathrm{E}$ was rescued after transfection of full-length in 
vitro transcripts of the cloned $\mathrm{HCoV} 229 \mathrm{E}$ cDNA into MRC-5 cells (Thiel et al. 2001a). Alternatively, the rescue of recombinant IBV has been reported by transfecting full-length coronavirus IBV cDNA into chick kidney (CK) cells that had been infected by a recombinant fowlpox virus, rFPV-T7 (Casais et al. 2001). The fowlpox virus mediates the expression of the bacteriophage T7 RNA polymerase. In contrast to the transfection of infectious HCoV 229E RNA, the rescue of recombinant IBV required the directed expression of the IBV nucleocapsid protein [mediated by transfection of a expression plasmid encoding the IBV nucleocapsid (N) protein]. This observation led us to develop a line of BHK cells which express the $\mathrm{HCoV} 229 \mathrm{E} \mathrm{N}$ protein, and, indeed, we found that the expression of this protein also facilitates the rescue of recombinant $\mathrm{HCoV}$ $229 \mathrm{E}$ coronaviruses after the transfection of cells with full-length in vitro transcripts.

\subsection{1 \\ Rescue of Recombinant Coronaviruses Using Full-Length In Vitro Transcripts}

The overall strategy to recover recombinant human coronavirus from vaccinia virus vHCoV-inf-1 genomic DNA is illustrated in Fig. 3. The full-length $\mathrm{HCoV} 229 \mathrm{E}$ cDNA is cloned downstream of a bacteriophage T7 RNA polymerase promoter, and a ClaI restriction endonuclease recognition sequence is located downstream of a synthetic poly(A) sequence, representing the $3^{\prime}$ end of the $\mathrm{HCoV}$ genome. Genomic vHCoVinf-1 DNA was prepared from purified recombinant vaccinia virus stocks and cleaved with ClaI enzyme. This DNA was then used as template to transcribe, in vitro, a capped RNA corresponding to the $\mathrm{HCoV}$ genome with bacteriophage T7 RNA polymerase. When this RNA was transfected into MRC-5 cells by lipofection, cytopathic effects indicative of human coronavirus infection developed throughout the culture after 6-7 days. A virus, designated $\mathrm{HCoV}$-inf-1, was rescued from the tissue culture supernatant, plaque purified and propagated to produce stocks containing approximately $1 \times 10^{7}$ TCID $50 / \mathrm{ml}$. Phenotypic analysis revealed that the growth kinetics, cytopathic effect and stability of $\mathrm{HCoV}$-inf- 1 were indistinguishable from those of parental virus. Furthermore, Northern hybridization analysis of poly(A)-containing RNA isolated from infected MRC-5 cells demonstrated that the patterns of viral genomic and subgenomic RNAs of HCoV-inf- 1 and $\mathrm{HCoV}$ 229E were identical. To confirm that, indeed, a recombinant virus had been rescued, the presence of a marker mutation which was introduced into the recombinant $\mathrm{HCoV}$ 

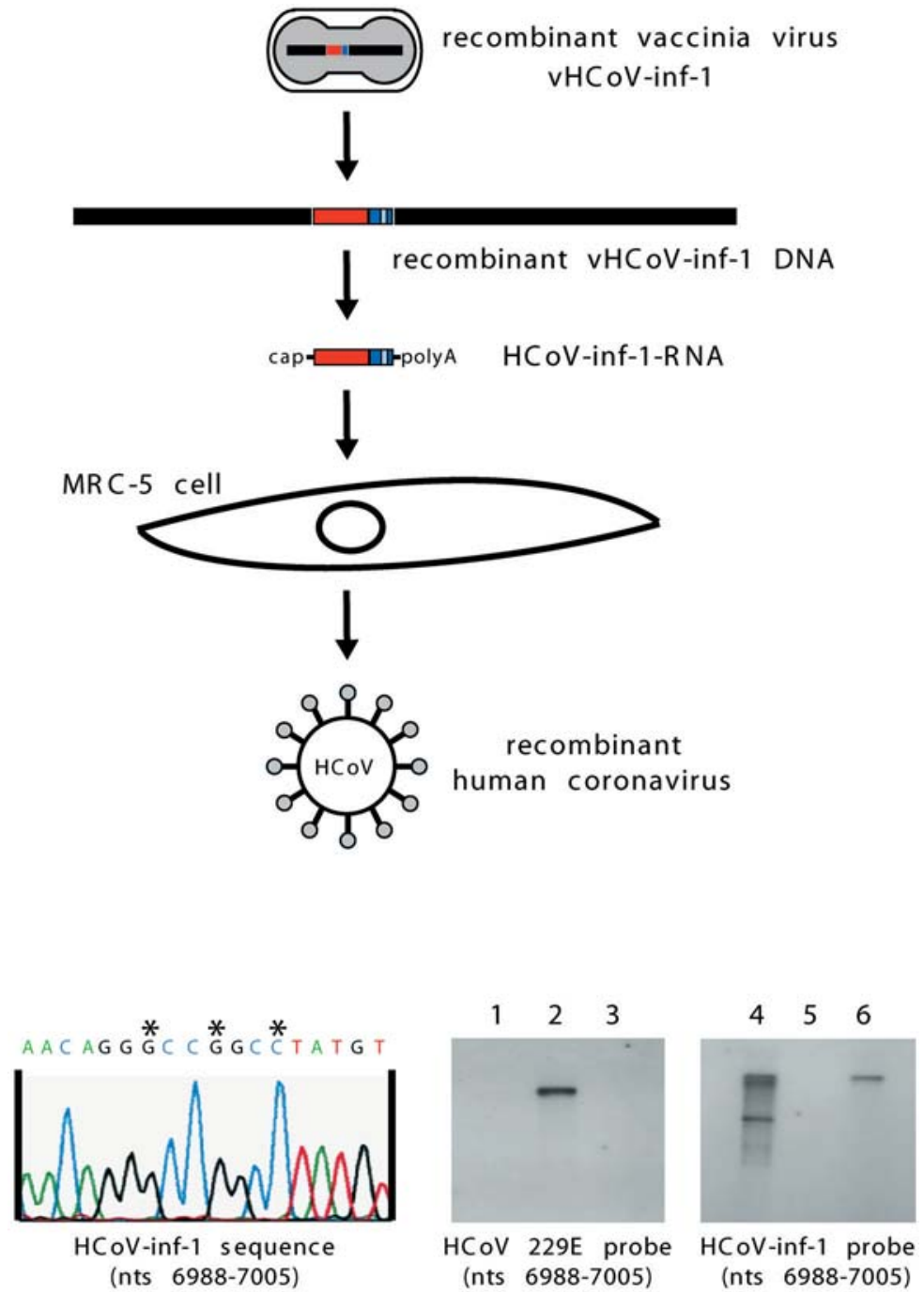

Fig. 3. Rescue of recombinant $\mathrm{HCoV} 229 \mathrm{E}$ from cloned, full-length cDNA. To recover recombinant $\mathrm{HCoV} 229 \mathrm{E}, 5^{\prime}$-capped RNA transcripts are produced in vitro with bacteriophage T7 RNA polymerase and $\mathrm{vHCoV}$-inf-1 genomic DNA as template. The transcripts are transfected into MRC-5 cells. The recombinant human coronavirus HCoV-inf-1 contains marker mutations $\left({ }^{*}\right)$ that are evident in the sequence of an RT-PCR product of poly(A)-containing RNA from HCoV-inf-1-infected cells. Also shown is a Northern hybridization analysis of in vitro transcribed HCoV-inf-1 RNA (lanes 1 and 4) and poly(A) containing RNA from parental HCoV 229E-infected MRC-5 cells (lanes 2 and 5) and HCoV-inf-1-infected MRC-5 cells (lanes 3 and 6). 
cDNA during the cloning procedure was analysed. As shown in Fig. 3, RT-PCR sequencing analysis of the relevant region of the HCoV-inf- 1 genome demonstrates that three nucleotides (nt 6,994, 6,997 and 7,000) were, as predicted, found to be changed compared to the HCoV 229E sequence. These results showed, conclusively, the rescue of recombinant human coronavirus and demonstrated that the coronavirus genomic RNA alone is able to initiate a productive infectious cycle.

\subsection{2}

\section{Rescue of Recombinant Coronavirus Using Full-Length cDNA}

Initially, the rescue of recombinant IBV from cloned cDNA was attempted by generating full-length T7-driven in vitro transcripts by using SalIrestricted vNotI/IBV ${ }_{\mathrm{FL}}$ genomic DNA as a template followed by transfection of the RNA into susceptible chick kidney (CK) cells (Casais et al. 2003). Although essentially the same protocol had been used successfully for the rescue of recombinant $\mathrm{HCoV} 229 \mathrm{E}$, the amounts and purity of full-length synthetic IBV RNA varied and attempts to rescue recombinant IBV from CK cells were not successful. Therefore, an alternative strategy was used (Fig. 4). First, CK cells were infected with rFPV-T7 to provide cytoplasmic bacteriophage T7 RNA polymerase (Britton et al. 1996). At $1 \mathrm{~h}$ after vFPV-T7-infection the cells were transfected with

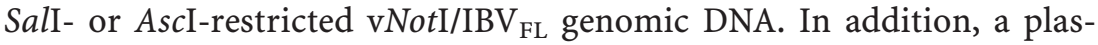
mid DNA which mediates the expression of the IBV nucleocapsid $(\mathrm{N})$ protein was co-transfected. When the infected/transfected cells developed cytopathic effects, the supernatant was filtered to remove vFPV-T7 virus and passaged on fresh CK cell monolayers until cytopathic effects characteristic of IBV infection were observed. Further analysis, including Northern blotting, immunofluorescence and finally RT-PCR sequencing to detect marker mutations, confirmed that recombinant IBV had been rescued. This result demonstrates that a reverse genetic system for IBV has been established based on cloning of full-length IBV cDNA

The RNAs were probed with a parental HCoV 229E-specific (lanes 1-3) or an HCoVinf-1-specific radiolabelled oligonucleotide (lanes 4-6) corresponding to the genomic nucleotides $6988-7005$, respectively. The HCoV 229E-specific probe hybridized to the HCoV 229E genomic RNA but not to recombinant HCoV-inf-1 genomic RNA. In contrast, the $\mathrm{HCoV}$-inf-1-specific probe hybridized to the HCoV-inf-1 genomic RNA and not to the parental HCoV 229E genomic RNA 


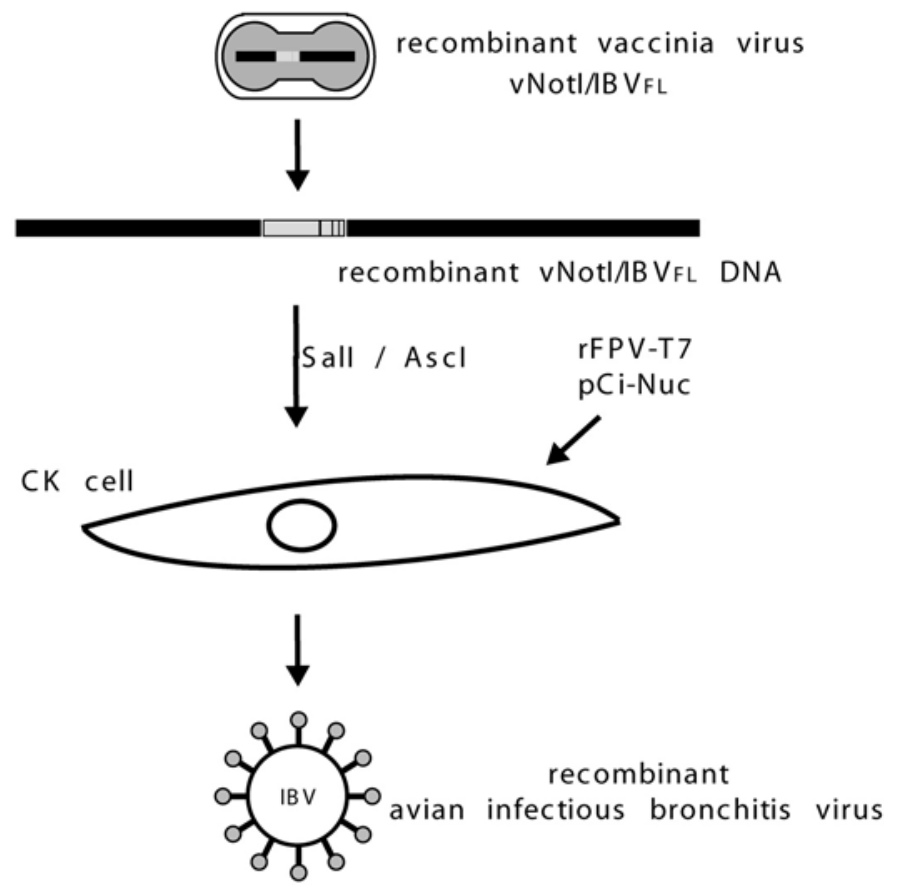

Fig. 4. Rescue of recombinant IBV from cloned, full-length cDNA. To recover recombinant IBV, SalI- or AscI-restricted genomic DNA of the recombinant vaccinia virus vNotI/IBV $_{\mathrm{FL}}$ (containing the full-length IBV cDNA) was transfected into chick kidney (CK) cells. The full-length IBV RNA is produced in vivo with a recombinant fowlpox virus rFPV-T7 which expresses the bacteriophage T7 RNA polymerase. In addition, the CK cells are transfected with a plasmid which mediates the expression of the IBV nucleocapsid protein (pCi-Nuc)

in a vaccinia virus vector. It should be noted that this procedure obviated the need to prepare purified vNotI/IBV $\mathrm{FL}$ genomic DNA for in vitro transcription and therefore represents an attractive alternative to rescue recombinant coronaviruses from cloned cDNA.

\subsection{3}

\section{Expression of the Nucleocapsid Protein Facilitates the Rescue of Recombinant Coronaviruses}

As described above, the rescue of recombinant IBV using in vivo transcription of full-length IBV RNA was dependent on the co-transfection of a plasmid DNA mediating the expression of the IBV N protein. A sim- 
ilar observation was made for the recovery of recombinant TGEV by Yount et al. (Yount et al. 2000). In contrast, the rescue of recombinant HCoV 229E and MHV-A59 (Coley et al., manuscript in preparation) and the rescue of recombinant TGEV with the BAC system were achieved without the directed expression of $\mathrm{N}$ protein. These data indicate that the coronavirus $\mathrm{N}$ protein is not absolutely required to establish a productive coronavirus infection with infectious RNA or DNA. However, it clearly facilitates rescue of recombinant viruses. Consequently, we have made two changes in our protocol for the rescue of recombinant coronaviruses with full-length in vitro transcripts.

First, we have used BHK-21 cells as target cells for the delivery of in vitro transcribed coronavirus genomic RNA by electroporation. On the one hand, BHK-21 cells can be efficiently transfected with RNA by electroporation, and at the same time, our data suggest that they are permissive for the production of, at least, HCoV 229E and MHV-A59. Second, to provide directed expression of the coronavirus $\mathrm{N}$ protein, we have produced stable BHK-21-derived cell lines which express coronavirus $\mathrm{N}$ proteins. An $\mathrm{HCoV} 229 \mathrm{E} \mathrm{N}$ protein-expressing cell line, designated $\mathrm{BHK}-\mathrm{HCoV}-\mathrm{N}$, has been produced for the $\mathrm{HCoV} 229 \mathrm{E}$ reverse genetic system, and an MHV N protein-expressing cell line, designated BHK-MHV-N, has been produced for the MHV-A59 reverse genetic system. Both cell lines are based on the Tet/ON expression system (Gossen et al. 1995) and thus allow the controlled expression of the respective $\mathrm{N}$ proteins on induction with doxycyclin. Using the BHK-HCoV-N cell line, we observed that the rescue of recombinant $\mathrm{HCoV} 229 \mathrm{E}$ after electroporation of full-length infectious HCoV RNA is greatly facilitated. The titres of virus in the supernatant of BHK-HCoV-N cells 3 days after transfection were about $1 \times 10^{4}$ TCID50/ml. Moreover, the use of the N-expressing cell line has enabled us to monitor coronavirus RNA synthesis by Northern blot analysis directly in transfected cells. Similar results have been obtained using the BHK-MHV-N cell line for the rescue of recombinant MHV-A59. It should be noted that in addition to the rescue of recombinant coronaviruses, the $\mathrm{N}$-expressing $\mathrm{BHK}$ cell lines also facilitate the analysis of coronavirus vector RNAs (see below).

\section{3 \\ Recombinant Coronaviruses}

Recombinant coronaviruses generated by the reverse genetic systems described above have been used in the analysis of coronavirus polypro- 
tein processing, the generation of chimeric coronaviruses which might be useful as coronavirus vaccines and the analysis of coronavirus pathogenesis.

\section{1}

\section{Analysis of HCoV 229E Replicase Polyprotein Processing}

The analysis of replication, transcription and polyprotein processing in many positive-strand RNA viruses has been facilitated by the development of reverse genetic systems based on cloned cDNA. The reverse genetic system for coronaviruses enables the generation and analysis of recombinant coronaviruses which carry mutations of choice. Specifically, it is now possible, for the first time, to generate and analyse recombinant coronaviruses which contain genetically modified replicase genes.

The HCoV 229E replicase gene is comprised of two overlapping open reading frames (ORFs), ORF1a and ORF1b, which extend about $20 \mathrm{~kb}$ from the $5^{\prime}$ end of the genome. It encodes two large polyproteins, ppla
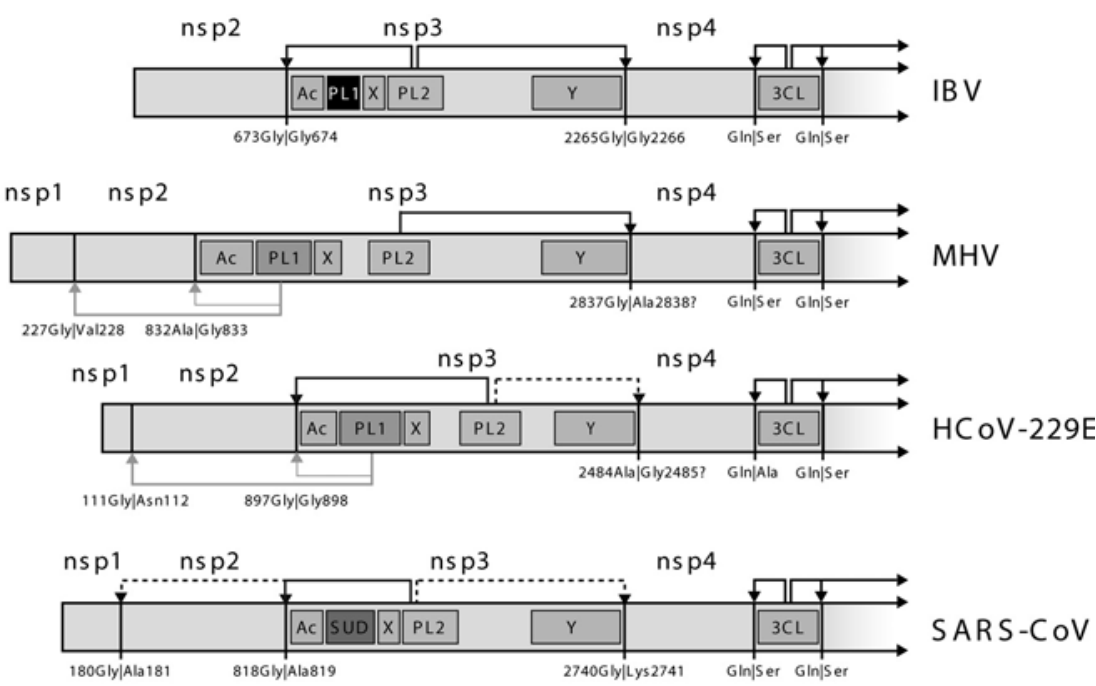

Fig. 5. Proteolytic processing of the amino-proximal regions of coronavirus replicase polyproteins. The amino-proximal regions of IBV, MHV, HCoV 229E and SARS$\mathrm{CoV}$ replicase polyproteins are illustrated. Processing events mediated by the papain-like and main proteinases are indicated (for details see the chapter by Ziebuhr, this volume). Abbreviations: $A c$, acidic domain; $P L 1$, papain-like proteinase 1; $S U D$, SARS-CoV unique domain; $X$, adenosine diphosphate-ribose $1^{\prime \prime}$-phosphatase; $P L 2$, papain-like proteinase 2; $Y$, nsp3 C-terminal domain; 3CL, 3C-like proteinase 
and pplab, which are extensively processed by the virus main proteinase, $\mathrm{M}^{\text {pro }}$, and two accessory papain-like proteinases, PL1 ${ }^{\text {pro }}$ and PL2 $2^{\text {pro }}$ (Ziebuhr et al. 2000). The N-proximal region of the polyproteins is processed by PL1 ${ }^{\text {pro }}$ and PL2 ${ }^{\text {pro }}$ to release three proteins, nsp1 $(9 \mathrm{kDa})$, nsp2 $(87 \mathrm{kDa})$ and nsp3 $(177 \mathrm{kDa})$ and, in addition, the N-terminus of nsp4 $(54 \mathrm{kDa})$ (Fig. 5). In vitro studies of the $\mathrm{HCoV} 229 \mathrm{E}$ polyprotein processing events revealed that PL1 ${ }^{\text {pro }}$ could cleave between nsp1 and nsp2 (Herold et al. 1998). Furthermore, it has been shown that PL1 ${ }^{\text {pro }}$ and $\mathrm{PL} 2^{\text {pro }}$ can cleave between nsp2 and nsp3, indicating overlapping substrate specificities. PL2 ${ }^{\text {pro }}$ cleaves this site efficiently, whereas PL1 ${ }^{\text {pro }}$ mediated cleavage is slow and significantly suppressed by proteolytic inactive PL2 ${ }^{\text {pro }}$ (Ziebuhr et al. 2001).

The in vitro data described above suggest a redundancy of $\mathrm{PL}^{\text {pro }}$ activities and raised the question of whether both HCoV 229E PL ${ }^{\text {pro }}$ activities are indeed required for virus replication. Thus, to gain a better understanding of the physiological roles of coronavirus papain-like proteinases, we have applied our reverse genetic system to the characterization of $\mathrm{HCoV} 229 \mathrm{E} \mathrm{PL1}{ }^{\text {pro }} / \mathrm{PL}_{2}{ }^{\text {pro }}$ in virus replication. Based on the recombinant vaccinia virus vHCoV-inf- 1 , harbouring the full-length $\mathrm{HCoV}$ cDNA, we have introduced nucleotide changes resulting in substitutions of the active-site cysteine residues of PL1 ${ }^{\text {pro }}$ (Cys1054Ala) and PL2 ${ }^{\text {pro }}$ (Cys1701Ala), respectively. Genomic DNA from the resulting vaccinia virus clones were used as template for T7-driven in vitro transcription to generate full-length $\mathrm{HCoV}-\mathrm{PL} 1(-)$ and $\mathrm{HCoV}-\mathrm{PL} 2(-)$ RNAs, respectively. As shown in Fig. 6 virus-specific RNAs were only detectable in HCoV-PL1(-) RNA-transfected BHK-HCoV-N cells. After transfer of the supernatant to MRC-5 cells only mutant viruses containing the $\mathrm{Cy}$ s1054Ala nucleotide change (at the PL1 ${ }^{\text {pro }}$ active site) could be rescued. In contrast, no mutant virus could be rescued if the supernatant of HCoV-PL2(-) RNA-transfected cells was transferred to MRC-5 cells. This result indicates that an active PL1 ${ }^{\text {pro }}$ enzyme is not required for HCoV 229E RNA synthesis and virus replication. However, the recombinant $\mathrm{HCoV}-\mathrm{PL} 1(-)$ virus displayed reduced growth kinetics, and reversion of mutant virus to the parental sequence at the PL1 ${ }^{\text {pro }}$ active site (reversion Ala1054Cys) occurred within a few passages on MRC-5 cells. The growth kinetics and RNA synthesis of the reverted virus were indistinguishable from those of the parental $\mathrm{HCoV} 229 \mathrm{E}$ virus. This indicates that although an active PL1 ${ }^{\text {pro }}$ enzyme is not absolutely required for virus replication it is still beneficial in terms of virus fitness. 
A

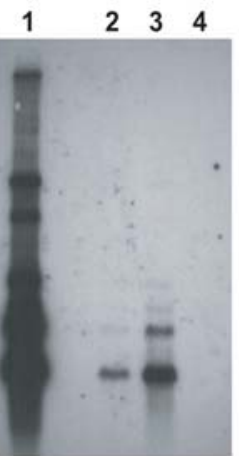

B

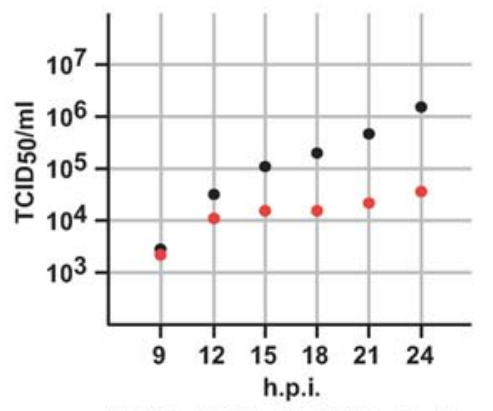

$\bullet \mathrm{HCoV} 229 \mathrm{E} \bullet \mathrm{HCoV}$ PL 1-minus

C

HCoV PL 1* RNA

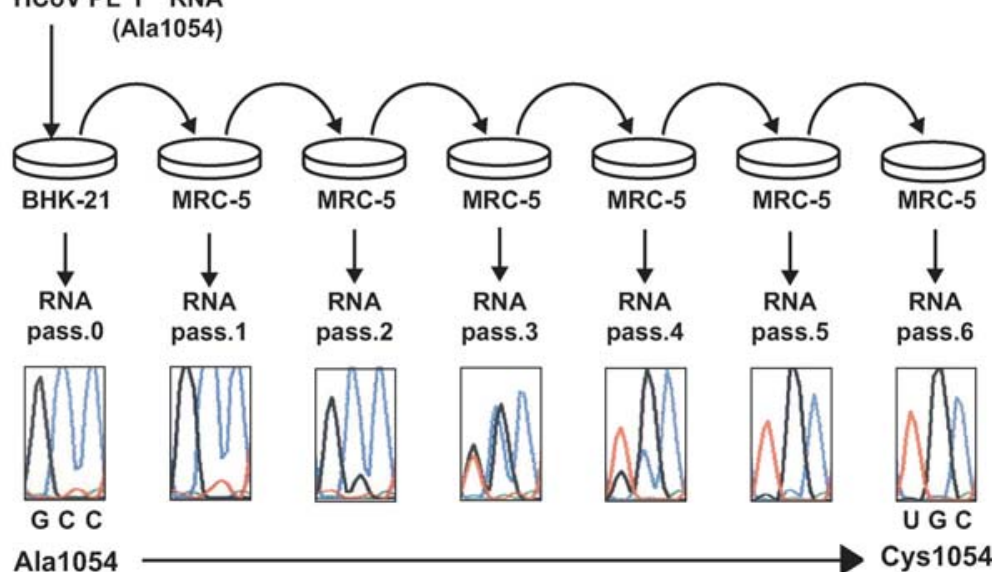

Fig. 6A-C. Rescue of recombinant HCoV 229E ablated for PL1 ${ }^{\text {pro }}$ activity. A Northern blot analysis of poly(A)-containing RNA from BHK-21 cells which have been transfected with in vitro synthesized HCoV-inf-1 RNA (lane 2), HCoV-PL1(-) RNA (lane 3) or HCoV-PL2(-) RNA (lane 4). Poly(A)-containing RNA from HCoV 229Einfected MRC-5 cells was used as a marker. B Growth kinetics of parental HCoV 229E and recombinant HCoV-PL1(-) determined after infection of MRC-5 cells (moi=1). C The reversion of the PL1 ${ }^{\text {pro }}$ active site mutation (GCC; Ala1054) of HCoV-PL1(-) to the parental sequence (UGC; Cys1054) during 6 passages on MRC-5 cells is illustrated 


\section{2}

\section{Analysis of IBV Spike Chimeras}

IBV is an important veterinary pathogen and, in common with other coronaviruses, the virus surface glycoprotein is thought to be an important determinant of cell tropism. To test this, the vaccinia virus-based coronavirus reverse genetic system has been used to produce a recombinant IBV in which the ectodomain region of the spike gene from IBV M41-CK replaced the corresponding region of the IBV Beaudette genome (Casais et al. 2003). Analysis of the recombinant IBV BeauR$\mathrm{M} 41(\mathrm{~S})$ showed that it had acquired the same growth characteristics and cell tropism as IBV M41-CK in vitro. These results demonstrate that the IBV spike glycoprotein is a determinant of cell tropism and, importantly, they show that the reverse genetic system can be used to generate recombinant coronaviruses with a precisely modified genome which may be used as vaccine strains.

\section{3}

\section{Recombinant MHV Is Fully Pathogenic in Mice}

$\mathrm{MHV}$ is the prototype of the class II coronaviruses, a group that also includes the SARS coronavirus. Moreover, MHV is the most experimentally accessible coronavirus system. For example, MHV-A59 replicates to high titres in cell culture and there exists a collection of temperature-sensitive mutants which are defective in the synthesis of viral RNA (Siddell et al. 2001). These features should facilitate the analysis of coronavirus RNA synthesis and aid in the elucidation of functions associated with coronavirus replicase proteins. Furthermore, the natural host of $\mathrm{MHV}$ is the mouse. There is a wealth of genetic and immunological information relating to inbred mouse strains, and there are an increasing number of transgenic mice strains which are ablated or defective in the expression of functional host cell genes, particularly those encoding proteins related to the immunological response to virus infection. MHV infection, therefore, is an ideal tool to study both the innate and adaptive immune responses to viruses. Finally, there are a number of informative animal disease models based on MHV infection, including models of virus-related demyelination and viral hepatitis (Haring and Perlman 2001). These models have provided, and will continue to provide, insights into the pathogenesis of virus infections. Recently, we have established a vaccinia virus-based reverse genetic system for MHV-A59 (Coley et al., manuscript in preparation). Our preliminary results indi- 


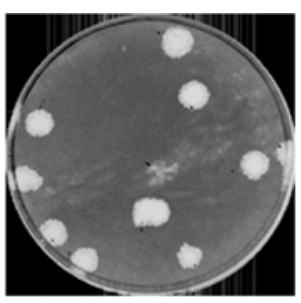

MHV A59

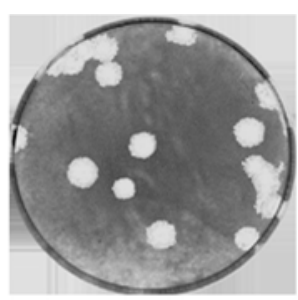

MHV-inf-1

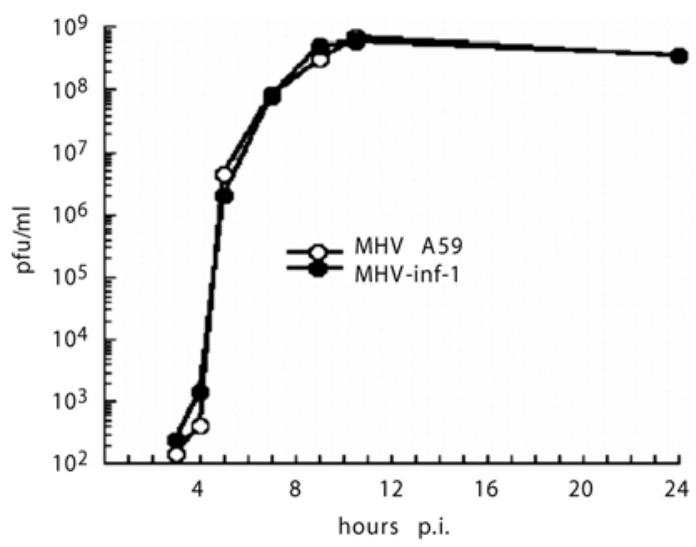

Fig. 7. Plaque morphology and growth kinetics of recombinant MHV-inf-1 generated from cloned full-length cDNA. Recombinant MHV was rescued, plaque purified and propagated by a single passage in murine 17 clone 1 cells. The stock was titrated by plaque assay on 17 clone 1 cells. The photographs show the $10^{-8}$ dilution of both MHV-A59 (strain OH99) and recombinant MHV-inf-1. The replication kinetics of MHV-A59 and MHV-inf- 1 in 17 clone 1 cells were also found to be identical

cate that the recombinant MHV-A59 replicates in cell culture to the same titre and with the same plaque morphology as laboratory-adapted strains of MHV-A59 (Fig. 7). Also, the recombinant virus replicates to comparable titres in mouse tissues, has approximately the same virulence as non-recombinant MHV-A59 and produces the same histopathological changes in the brains and liver of infected mice (Coley et al., manuscript in preparation). 


\section{4 \\ Generation of Replicon RNAs}

The development of reverse genetic systems for coronaviruses provides an opportunity to carry out an extensive biological characterization of the viral replicative proteins and functions. In addition to the analysis of recombinant coronaviruses, replicative functions can also be studied in non-infectious systems. For example, the concept of using autonomously replicating RNAs (replicon RNAs) has been explored in a number of positive-strand RNA virus systems and has greatly facilitated the functional analysis of viral replication and transcription (Pietschmann and Bartenschlager 2001; Westaway et al. 2003). Furthermore, selectable replicon RNAs that carry a marker for selection in cell culture have been developed in order to generate stable, replicon RNA-containing cell lines. Such cell lines have been used to study replicative functions and are of particular interest if a virus cannot be efficiently propagated in tissue culture, e.g. hepatitis C virus. Because no structural genes and, therefore, no infectious viruses are formed, replicon-based systems also represent an attractive tool for the analysis of replicative functions if the pathogenicity of the virus is a concern.

\section{1}

\section{Replicase Gene Products Suffice for Discontinuous Transcription}

For most positive-strand RNA viruses, the replicase gene and the $5^{\prime}$ - and $3^{\prime}$-genomic termini suffice for autonomous replication of the viral RNA. It has been demonstrated that, indeed, the presence of $5^{\prime}$ - and $3^{\prime}$-genomic termini of coronavirus genomes is necessary for efficient replication of defective RNAs in helper virus-infected cells (Kim et al. 1993). The availability of reverse genetic systems now allows us to analyse the role of the replicase gene products in coronavirus replication and transcription. Based on the full-length $\mathrm{HCoV} 229 \mathrm{E}$ cDNA, cloned in a vaccinia virus vector, an RNA has been constructed that contains the $5^{\prime}$ and $3^{\prime}$ ends of the genomic RNA, the entire replicase gene, and a single reporter gene (encoding for GFP) (Fig. 8A; Thiel et al. 2001b). The GFP gene has been cloned downstream of the replicase gene and a transcription regulatory sequence (TRS) to enable the synthesis of a subgenomic mRNA encoding GFP. When this RNA was transfected into BHK-21 cells, a small percentage of cells displayed green fluorescence, indicative for GFP expression. After isolation of poly(A)-containing RNA from BHK-transfected cells an RT-PCR product could be identified which represents the lead- 
A

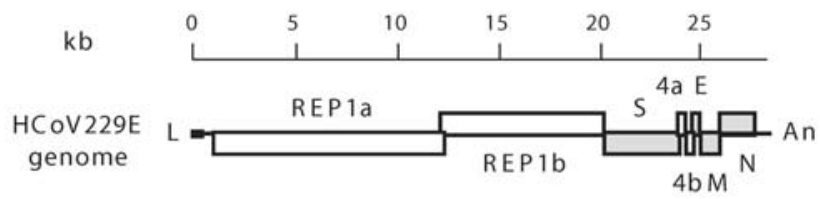

vec-1

RNA

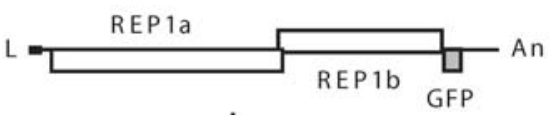

B HK-21 cell
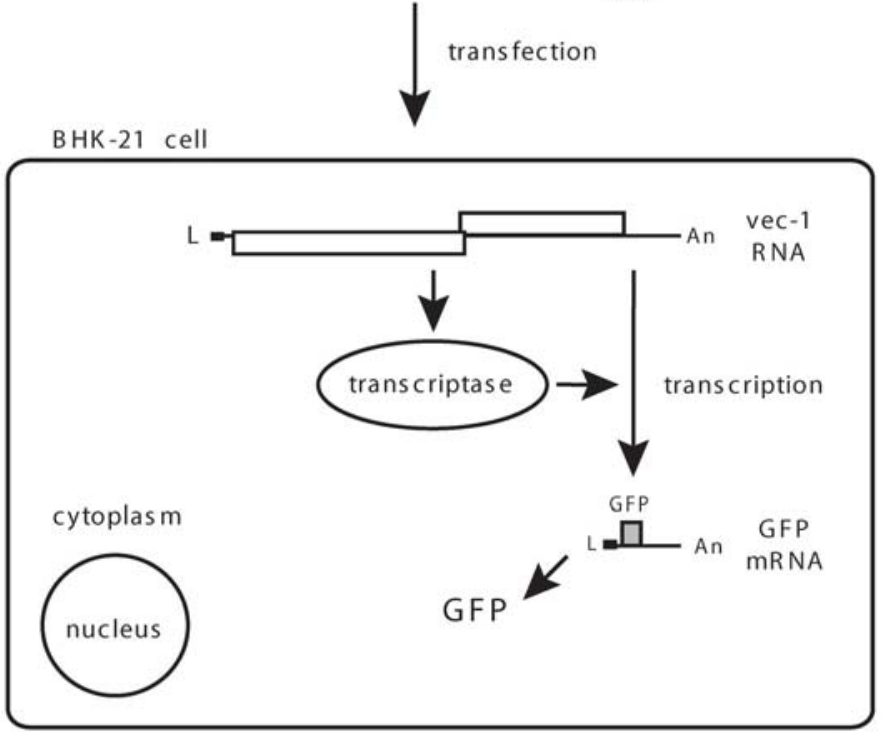

B

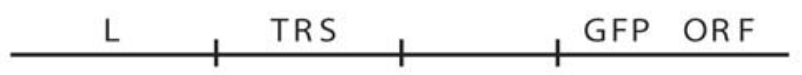

GUCUACUU UUCUCAACUAAAUAAC AUGG UGAGCA

GTCTACT TT TCTCAACTAAATAACATGGTGAGCA

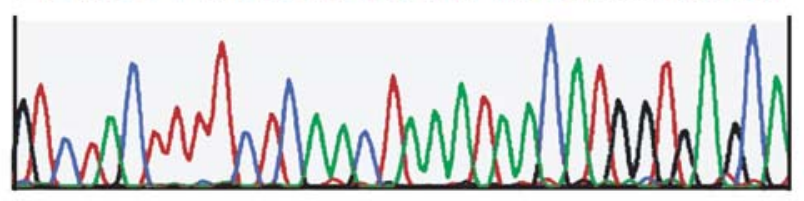

RT-PCR sequencing of GFP mRNA 
er-body junction of a subgenomic mRNA encoding GFP. Sequence analysis of this RT-PCR product showed that coronavirus-specific, subgenomic mRNA synthesis has occurred and the leader-body fusion has taken place at the expected position (Fig. 8B). These results demonstrate conclusively that the coronavirus replicase gene products are the only viral proteins needed to assemble a functional complex capable of discontinuous transcription. However, it should be noted that these results do not prove that replication of the transfected RNA has occurred, nor do they exclude the possibility that additional viral or host cell proteins may have regulatory roles in coronavirus replication or transcription. It is striking in this respect that only a small percentage $(\sim 0.1 \%)$ of green fluorescent cells could be observed after RNA transfection into BHK-21 cells. In a similar experiment, $\mathrm{HCoV} 229 \mathrm{E}-$ based vector RNA has been transfected into BHK-21 cells together with a synthetic mRNA encoding for the HCoV 229E N protein. In this case about 3\% of green fluorescent cells could be observed (Thiel et al. 2003). This result again indicates that the coronavirus $\mathrm{N}$ protein might be involved (directly or indirectly) in the (regulation of) replication and/or transcription of viral RNA. Further studies are needed to address this issue and, clearly, reverse genetic approaches represent valuable tools to study the putative function(s) of coronavirus $\mathrm{N}$ proteins.

\section{2}

\section{Generation of Autonomously Replicating RNAs}

Although our analysis of replicon RNAs revealed the basic requirements for coronavirus discontinuous transcription, we were unable to demonstrate replication of coronavirus replicon RNAs in transfected cells. While using the reverse genetic system for the recovery of recombinant coronaviruses and during the development of coronavirus multigene RNA vectors, we have gained evidence that the nucleocapsid protein fa-

Fig. 8A, B. Replicase gene products suffice for coronavirus discontinuous transcription. A The structural relationship of the $\mathrm{HCoV} 229 \mathrm{E}$ genome, the in vitro-transcribed HCoV-vec-1 RNA and the intracellular mRNA produced by coronavirus transcriptase-mediated discontinuous transcription is illustrated. B The sequence of the transcription regulating sequence (TRS) region of the intracellular GFP mRNA was obtained from an RT-PCR product of poly(A)-containing RNA from HCoV-vec1-transfected cells. The sequences corresponding to the HCoV 229E leader $(L)$, the TRS region and the first $10 \mathrm{nt}$ of the GFP-ORF are shown 

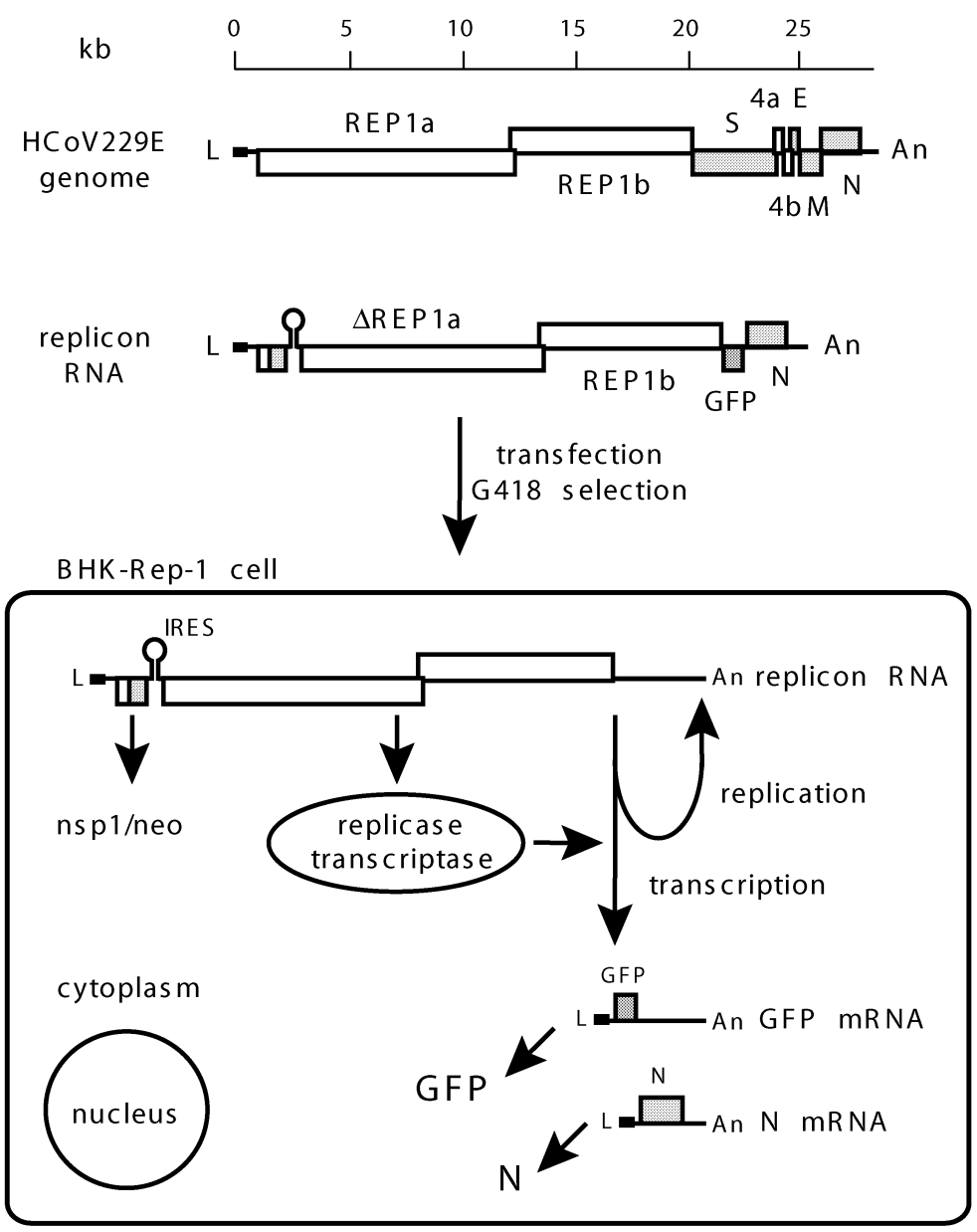

Fig. 9. Generation of stable cell lines containing autonomously replicating coronavirus RNA. The structural relationship of the HCoV 229E genome, the in vitro-transcribed replicon RNA and the intracellular mRNAs (GFP and N mRNAs) produced by coronavirus replicase/transcriptase-mediated discontinuous transcription is illustrated. The insertion of the neomycin resistance gene at the replicase nsp1/nsp2 junction allows the selection of stable cell lines with G418

cilitates the rescue of recombinant coronaviruses and, furthermore, increases the number of cells that contain an active transcription complex after electroporation of vector RNAs. Consequently, we constructed an RNA that contains the $5^{\prime}$ and $3^{\prime}$ ends of the HCoV 229E genome, the 
HCoV 229E replicase gene, the GFP reporter gene and the HCoV 229E nucleocapsid gene. In addition, to provide the basis for the selection of stable cell clones that contain autonomously replicating RNA, we have introduced a selectable marker gene into the replicase gene conferring resistance to neomycin (Fig. 9).

After transfection of this selectable replicon RNA into BHK-21 cells we were, indeed, able to select multiple cell clones containing an autonomously replicating RNA. These cells have been shown to stably maintain the replicating RNA and, moreover, display green fluorescence due to the replicon RNA-mediated GFP expression (Hertzig et al. 2004). This is the first example of coronavirus-derived RNAs which can be selected for replication in cell culture. The selectable replicon RNA, in combination with GFP reporter gene expression, provides an excellent basis to analyse the function of coronavirus replicase inhibitors in cell culture. Thus it is now possible to test the effects of antiviral compounds on coronavirus replication by simply seeding out the replicon RNA-containing cells and assaying for reporter gene expression levels. Decreasing reporter gene expression will indicate putative antiviral activity of a particular compound, which can then be tested for specificity and efficacy. Given the striking similarities of replicative enzymes and functions amongst coronaviruses, the currently available replicon RNA, based on $\mathrm{HCoV} 229 \mathrm{E}$, can already be used to screen for suitable drugs which target coronavirus infections, including SARS. In the long term, a SARS-CoV replicon RNA can be used to screen for SARS-specific inhibitors. The SARS-CoV replicon system will provide a versatile platform technology which allows the development of a high-throughput antiviral screening assay based on reporter gene expression. Furthermore, it circumvents biosafety concerns associated with SARS-CoV, because it represents a rapid, convenient and safe assay for SARS-CoV replication without the need to grow infectious SARS-CoV.

\section{5}

\section{Development of Coronavirus-Based Multigene Vectors}

The molecular biology of coronaviruses and the specific features of the human coronavirus 229E ( $\mathrm{HCoV} 229 \mathrm{E})$ system indicate that $\mathrm{HCoV}$ $229 \mathrm{E}-$ based vaccine vectors have the potential to become a new class of viral vaccines. First, the receptor for $\mathrm{HCoV} 229 \mathrm{E}$, human aminopeptidase N (hAPN or CD13) is expressed on human dendritic cells (DC) and macrophages, indicating that targeting of $\mathrm{HCoV} 229 \mathrm{E}-$ based vectors to 
professional antigen-presenting cells can be achieved by receptor-mediated transduction. Second, coronaviruses display a unique transcription mechanism resulting in the synthesis of multiple subgenomic mRNAs encoding mainly structural proteins. Because the structural genes can be replaced by multiple heterologous genes, these vectors represent safe, non-infectious vector RNAs. Third, it has been shown that HCoV 229E multigene vectors can be packaged to virus-like particles (VLPs) if the structural proteins are expressed in trans. Most interestingly, VLPs containing HCoV 229E-based vector RNA have the ability to transduce human DC and to mediate heterologous gene expression in these cells (Thiel et al. 2003). Thus the expression of multiple antigens in combination with specific DC tropism represents an unprecedented potential to induce both $\mathrm{T}$ cell and antibody responses against multiple antigens. Finally, coronavirus infections are mainly associated with respiratory and enteric diseases and natural transmission of coronaviruses occurs via mucosal surfaces. HCoV 229E infections are mainly encountered in children, and re-infection occurs frequently in adults. It is therefore unlikely that preexisting immunity against $\mathrm{HCoV} 229 \mathrm{E}$ will have a significant impact on the vaccination efficiency if $\mathrm{HCoV} 229 \mathrm{E}-$ based vectors are used in humans.

\section{1}

\section{Multigene Expression Using Coronavirus-Based Vectors}

With the reverse genetic systems available, it is now possible to make use of the unique characteristics of coronavirus transcription to develop coronavirus expression vectors. The rationale of expressing heterologous genes with coronavirus-mediated transcription is to insert a transcriptional cassette, comprised of a TRS located upstream of the gene of interest, into a coronavirus genome, minigenome or vector RNA. We have explored a vector RNA-based strategy using the HCoV 229E reverse genetic system and could show for human coronavirus vector RNAs that a region of at least $5.7 \mathrm{~kb}$ is dispensable for discontinuous transcription (Thiel et al. 2001b). This region contained all structural genes, and therefore our vector RNAs are not infectious. For the construction of coronavirus-based non-infectious, multigene vectors, we consider that about one-third of the genome, or up to $9 \mathrm{~kb}$, could be replaced by multiple transcriptional cassettes. Indeed, we could demonstrate that it is possible to construct a human coronavirus vector RNA capable of mediating the expression of multiple heterologous proteins, namely the chloramphenicol-acetyltransferase, the firefly luciferase and GFP 

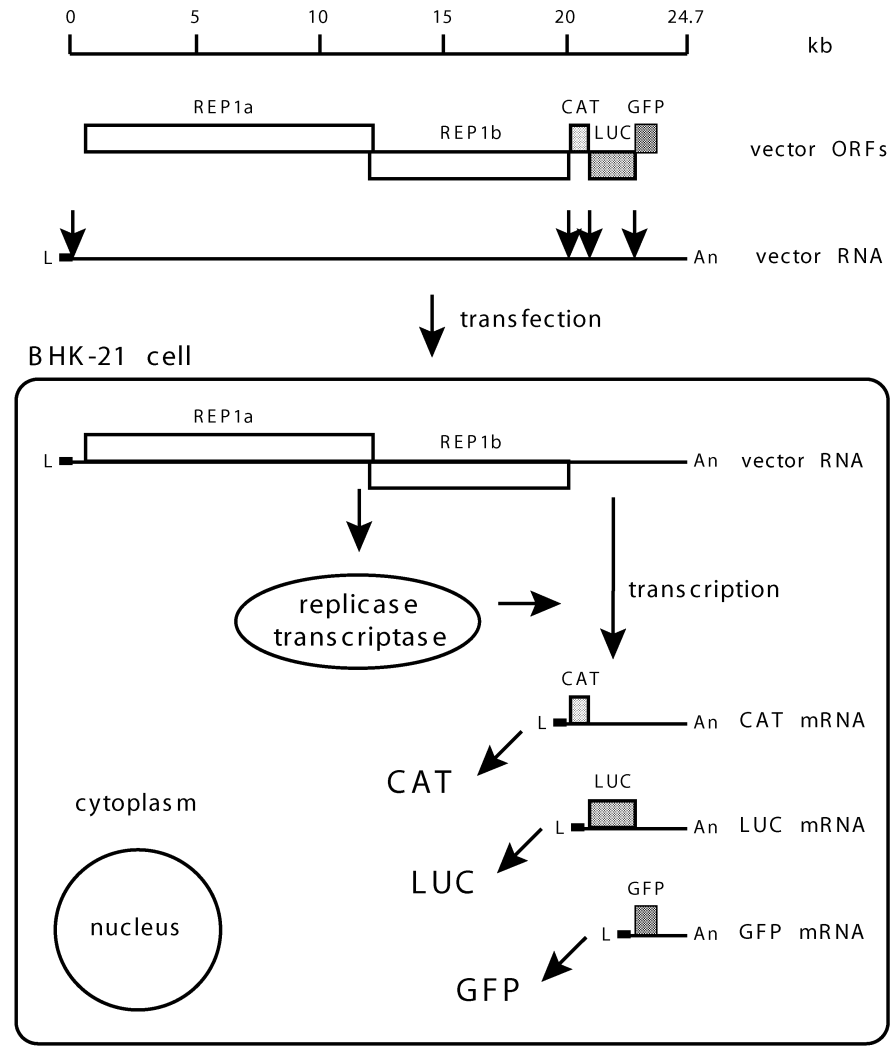

Fig. 10. Multigene RNA vectors based on coronavirus transcription. The structural relationship of HCoV-based vector ORFs, TRS elements (arrows) and the intracellular mRNAs produced by coronavirus-mediated transcription is illustrated, together with the intracellular translation products (i.e. the replicase/transcriptase, CAT, LUC and GFP)

(Fig. 10). These results indicate that coronavirus-based vector systems might be useful for heterologous gene expression, especially for longer and multiple genes.

\section{2}

\section{Coronavirus-Based Vectors as Potential Vaccines}

An important consideration for viral vaccine vectors is their potential for efficient delivery of their genetic material to specific target cells. For example, targeting of viral vaccine vectors to dendritic cells (DC) is 
highly desirable in order to optimize vaccine efficacy. In this respect, it is important to note that the $\mathrm{HCoV} 229 \mathrm{E}$ receptor, human aminopeptidase N (hAPN or CD13), is expressed at high levels on human DC (Summers et al. 2001). This implies that HCoV 229E-based VLPs could be used to efficiently (receptor-mediated uptake) transduce these cells. Indeed, it has been demonstrated that HCoV 229E-based VLPs can be used to transduce immature and mature human DC and that vector-mediated heterologous gene expression can be achieved in human DC (Thiel et al. 2003). Multigene vectors, based on HCoV 229E, represents a particularly promising tool to genetically deliver multiple genes such as tumour or HIV antigens and immunostimulatory cytokines to human DC.

Despite the remarkable potential of human coronavirus vectors as vaccines, the efficacy of coronavirus multigene vectors has not yet been demonstrated in vivo. For obvious reasons, a small animal model is desirable to address this issue. We have, therefore, established a reverse genetic system for MHV (see Sect. 3.3) which allows construction of MHV vector RNAs resembling their HCoV 229E counterparts. Murine DC can be infected with MHV, indicating that the analysis of recombinant MHV vectors in the context of a murine animal model may well serve as a

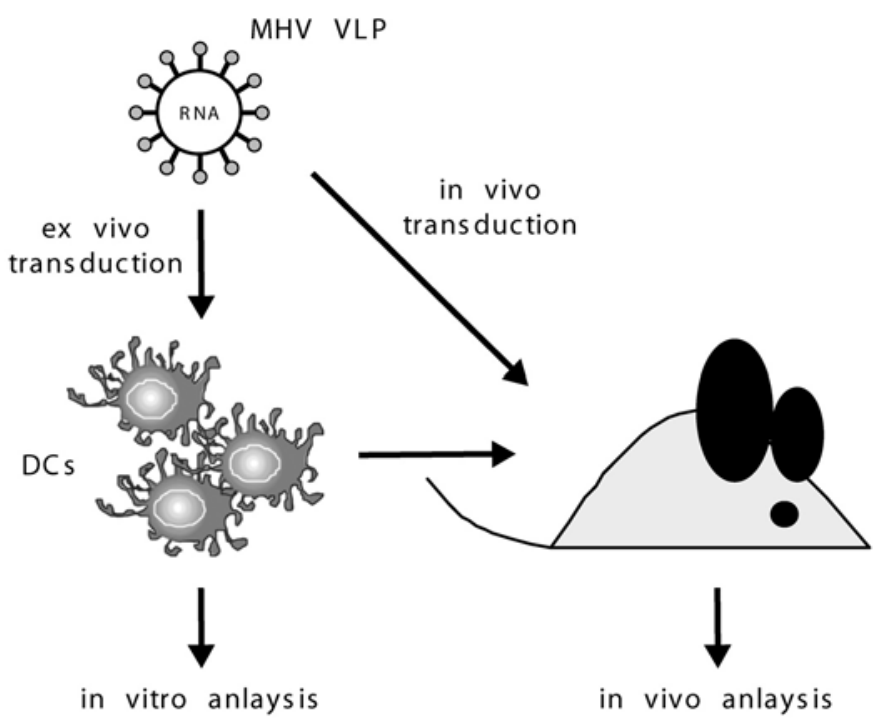

Fig. 11. A murine model to assess the efficacy of coronavirus-based multigene vaccine vectors 
paradigm for the development of coronavirus vaccine vectors (Fig. 11). In addition, the murine model will allow the use of inbred and transgenic mice and a variety of established immunological techniques which are indispensable for the analysis of vector-induced immune responses.

An important prerequisite to study of the efficacy of coronavirus vaccine vectors is the availability of VLPs that can be produced to high titres. Therefore, packaging cell lines must be established which mediate the expression of coronavirus structural proteins in trans. It has been shown that recombinant MHV defective-interfering particles can be produced in the absence of helper virus if the structural proteins are expressed in trans (Bos et al. 1996). Furthermore, a replication-competent but propagation-deficient TGEV vector RNA which lacks the E gene can be packaged when the E protein is expressed under the control of the cytomegalovirus promoter or by using alphavirus-based expression systems (Curtis et al. 2002; Ortego et al. 2002). Once an efficient coronavirus packaging strategy has been established, the efficacy of coronavirus multigene vaccine vectors can be assessed in vivo with the murine animal model.

\section{6 \\ Discussion}

In the past, the study of coronavirus genetics was broadly restricted to the analysis of temperature-sensitive $(t s)$ mutants, the analysis of defective RNA templates which depend on replicase proteins provided by a helper virus and the analysis of mutant and chimeric viruses generated by targeted recombination. Each of these methods produced valuable information, but, in one way or another, each has its own limitations. For example, the generation of $t s$ mutants is essentially a random process and a great deal of effort must be expended to produce a comprehensive collection of mutants representing the possible complementation groups or cistrons encoded in the coronavirus genome. Similarly, targeted recombination is a powerful tool for the generation of site-specific coronavirus mutants and chimeras. However, for technical reasons, it is restricted to the $3^{\prime}$ one-third of the genome, encoding the structural and accessory proteins. So the development of reverse genetic approaches which do not have these limitations should provide a tremendous impetus to the study of coronavirus replication and biology. We predict that the new reverse genetic systems, including the one described here, will be used very quickly to study the structure and function of coronavirus 
replicase genes and genes that are non-essential for replication in cell culture but clearly provide a selective advantage in vivo. We also predict that the reverse genetic systems will lead to the development of sophisticated RNA replicon systems which will be used as vectors for the delivery of heterologous genes in gene therapy and as biosafe diagnostic tools for the identification of, for example, coronavirus replicase inhibitors.

\section{References}

Almazan F, Gonzalez JM, Penzes Z, Izeta A, Calvo E, Plana-Duran J, Enjuanes L (2000) Engineering the largest RNA virus genome as an infectious bacterial artificial chromosome. Proc Natl Acad Sci USA 97:5516-5521

Ball LA (1987) High-frequency homologous recombination in vaccinia virus DNA. J Virol 61:1788-1795

Bos EC, Luytjes W, van der Meulen HV, Koerten HK, Spaan WJ (1996) The production of recombinant infectious DI-particles of a murine coronavirus in the absence of helper virus. Virology 218:52-60

Britton P, Green P, Kottier S, Mawditt KL, Penzes Z, Cavanagh D, Skinner MA (1996) Expression of bacteriophage T7 RNA polymerase in avian and mammalian cells by a recombinant fowlpox virus. J Gen Virol 77:963-7

Casais R, Dove B, Cavanagh D, Britton P (2003) Recombinant avian infectious bronchitis virus expressing a heterologous spike gene demonstrates that the spike protein is a determinant of cell tropism. J Virol 77:9084-9089

Casais R, Thiel V, Siddell SG, Cavanagh D, Britton P (2001) Reverse genetics system for the avian coronavirus infectious bronchitis virus. J Virol 75:12359-12369

Curtis KM, Yount B, Baric RS (2002) Heterologous gene expression from transmissible gastroenteritis virus replicon particles. J Virol 76:1422-1434

Gossen M, Freundlieb S, Bender G, Muller G, Hillen W, Bujard H (1995) Transcriptional activation by tetracyclines in mammalian cells. Science 268:1766-1769

Haring J, Perlman S (2001) Mouse hepatitis virus. Curr Opin Microbiol 4:462-466

Herold J, Gorbalenya AE, Thiel V, Schelle B, Siddell SG (1998) Proteolytic processing at the amino terminus of human coronavirus $229 \mathrm{E}$ gene 1-encoded polyproteins: identification of a papain-like proteinase and its substrate. J Virol 72:910-918

Hertzig T, Scandella E, Schelle B, Ziebuhr J, Siddell SG, Ludewig B, Thiel V (2004) Rapid identification of coronavirus replicase inhibitors using a selectable replicon RNA. J Gen Virol 85:1717-1725

Kerr SM, Smith GL (1991) Vaccinia virus DNA ligase is nonessential for virus replication: recovery of plasmids from virus-infected cells. Virology 180:625-632

Kim YN, Jeong YS, Makino S (1993) Analysis of cis-acting sequences essential for coronavirus defective interfering RNA replication. Virology 197:53-63

Merchlinsky M, Moss B (1992) Introduction of foreign DNA into the vaccinia virus genome by in vitro ligation: recombination-independent selectable cloning vectors. Virology 190:522-526 
Ortego J, Escors D, Laude H, Enjuanes L (2002) Generation of a replication-competent, propagation-deficient virus vector based on the transmissible gastroenteritis coronavirus genome. J Virol 76:11518-11529

Pietschmann T, Bartenschlager R (2001) The hepatitis C virus replicon system and its application to molecular studies. Curr Opin Drug Discov Devel 4:657-664

Scheiflinger F, Dorner F, Falkner FG (1992) Construction of chimeric vaccinia viruses by molecular cloning and packaging. Proc Natl Acad Sci USA 89:9977-9981

Siddell S, Sawicki D, Meyer Y, Thiel V, Sawicki S (2001) Identification of the mutations responsible for the phenotype of three MHV RNA-negative ts mutants. Adv Exp Med Biol 494:453-458

Smith GL, Moss B (1983) Infectious poxvirus vectors have capacity for at least 25000 base pairs of foreign DNA. Gene Ther 25:21-28

Summers KL, Hock BD, McKenzie JL, Hart DN (2001) Phenotypic characterization of five dendritic cell subsets in human tonsils. Am J Pathol 159:285-295

Thiel V, Herold J, Schelle B, Siddell SG (2001a) Infectious RNA transcribed in vitro from a cDNA copy of the human coronavirus genome cloned in vaccinia virus. J Gen Virol 82:1273-1281

Thiel V, Herold J, Schelle B, Siddell SG (2001b) Viral replicase gene products suffice for coronavirus discontinuous transcription. J Virol 75:6676-6681

Thiel V, Karl N, Schelle B, Disterer P, Klagge I, Siddell SG (2003) Multigene RNA vector based on coronavirus transcription. J Virol 77:9790-9798

Westaway EG, Mackenzie JM, Khromykh AA (2003) Kunjin RNA replication and applications of Kunjin replicons. Adv Virus Res 59:99-140

Yount B, Curtis KM, Baric RS (2000) Strategy for systematic assembly of large RNA and DNA genomes: transmissible gastroenteritis virus model. J Virol 74:1060010611

Ziebuhr J, Snijder EJ, Gaorbalenya AE (2000) Virus-encoded proteinases and proteolytic processing in the Nidovirales. J Gen Virol 81:853-879

Ziebuhr J, Thiel V, Gorbalenya AE (2001) The autocatalytic release of a putative RNA virus transcription factor from its polyprotein precursor involves two paralogous papain-like proteases that cleave the same peptide bond. J Biol Chem 276:3322033232 